\title{
GLOBAL, GEOMETRICAL COORDINATES ON FALBEL'S CROSS-RATIO VARIETY
}

\author{
JOHN R. PARKER \& IOANNIS D. PLATIS
}

\begin{abstract}
Falbel has shown that four pairwise distinct points on the boundary of complex hyperbolic 2-space are completely determined, up to conjugation in $\mathrm{PU}(2,1)$, by three complex crossratios satisfying two real equations. We give global geometrical coordinates on the resulting variety.
\end{abstract}

\section{INTRODUCTION}

It is well known that a set of four pairwise distinct points on the Riemann sphere is determined up to Möbius equivalence by their cross-ratio. Moreover, permuting these points determines a new cross-ratio that may be expressed as a simple function of the first one; see section 4.4 of [1]. The cross-ratio was generalised to sets of four points in the boundary of complex hyperbolic space by Korányi and Reimann [7]. By simply counting dimensions, it is easy to see that this complex number cannot completely determine the four points up to $\mathrm{PU}(2,1)$ equivalence. By permuting the points we obtain 24 cross-ratios. There are certain relations between them; see [5] or [11]. After factoring out these relations, one is left with three complex cross-ratios satisfying two real relations; see [2]. Falbel's cross-ratio variety $\mathfrak{X}$ is the subset of $\mathbb{C}^{3}$ where these relations are satisfied. Falbel has shown in Proposition 2.4 of [2] that these three complex numbers uniquely determine our initial set of four points up to $\mathrm{PU}(2,1)$ equivalence and, moreover, it is not possible to merely use two of the cross-ratios to do this. He goes on to discuss cross-ratios in a much more general setting. We will not be concerned with this level of generality here.

In [9] we used points of $\mathfrak{X}$ in our generalisation of Fenchel-Nielsen coordinates to the complex hyperbolic setting. There it was more convenient to use a slightly different normalisation from that of Falbel. In this paper we maintain the notation of [9]. Thus, we take Falbel's cross-ratio variety $\mathfrak{X}$ to be parametrised by three complex numbers $\mathbb{X}_{1}, \mathbb{X}_{2}$ and $\mathbb{X}_{3}$ not equal to 0 or 1 and satisfying the following identities:

$$
\begin{aligned}
\left|\mathbb{X}_{3}\right| & =\left|\mathbb{X}_{2}\right| /\left|\mathbb{X}_{1}\right| \\
2\left|\mathbb{X}_{1}\right|^{2} \Re\left(\mathbb{X}_{3}\right) & =\left|\mathbb{X}_{1}\right|^{2}+\left|\mathbb{X}_{2}\right|^{2}-2 \Re\left(\mathbb{X}_{1}+\mathbb{X}_{2}\right)+1 .
\end{aligned}
$$

(These equations are equivalent to the equations in Proposition 2.3 of [2] under the substitution $\omega_{0}=1 / \mathbb{X}_{3}, \omega_{1}=1 / \mathbb{X}_{1}$ and $\omega_{2}=\overline{\mathbb{X}}_{2}$.) From this description the topology and the geometry of the cross-ratio variety are mysterious. The purpose of this paper is to use global geometrical coordinates to give an alternative description of $\mathfrak{X}$ that makes its topology somewhat more transparent. In [3] the differential geometric structure of $\mathfrak{X}$ is discussed in more detail. A further application of crossratios is their use in the generalisation of Jørgensen's inequality to complex hyperbolic space [6]. We can interpret those results geometrically using the coordinates developed here.

We shall assume some background knowledge of complex hyperbolic geometry. Further details concerning the wider context may be found in Goldman [5]. Specific details, including many of the conventions we use, are given in [9].

1991 Mathematics Subject Classification. 32G05,32M05.

IDP was supported by a Marie Curie Reintegration grant (Contract No.MEIF-CT-2005-028371) within the 6th Community Framework Programme. 


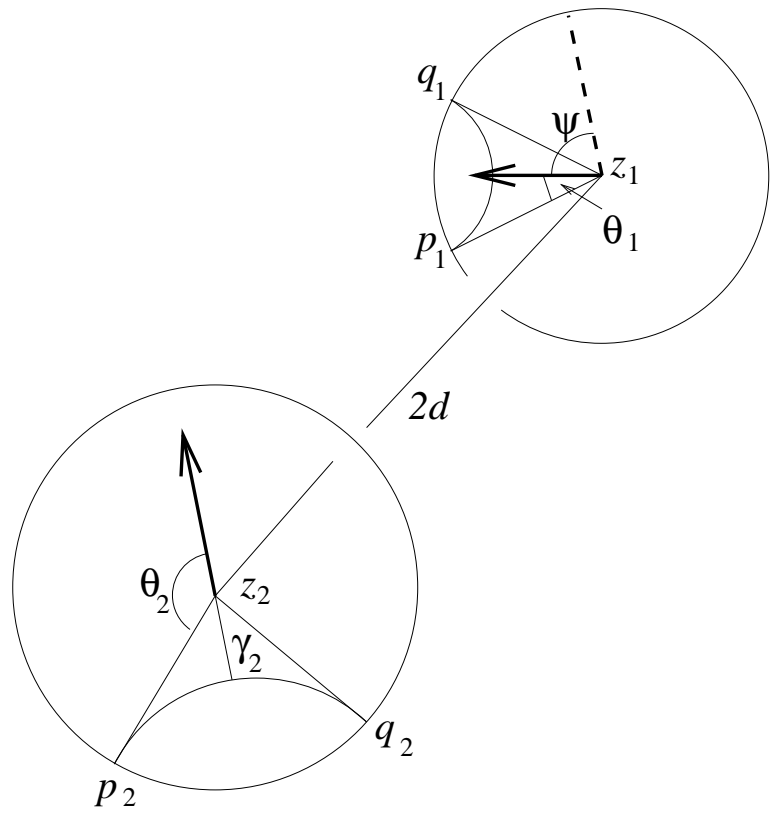

FiguRE 2.1. The parameters for a pair of ultra-parallel geodesics. Here $d>0$, $0<\theta_{1}<\pi / 2, \pi / 2<\theta_{2}<\pi, 0<\psi<\pi / 2$.

\section{THE MAIN THEOREMS}

Let $\langle\cdot, \cdot\rangle$ be a Hermitian form on $\mathbb{C}^{3}$ of signature $(2,1)$ and let $\mathbf{H}_{\mathbb{C}}^{2}$ be the image of its negative vectors under complex projection on $\mathbb{C}^{3}-\{0\}$; see [5]. Let $p_{1}, q_{1}, p_{2}, q_{2}$ be four pairwise distinct points in $\partial \mathbf{H}_{\mathbb{C}}^{2}$ (and so each of them may be lifted to a null vector in $\mathbb{C}^{3}-\{0\}$ ). Let $L_{i}$ denote the complex line spanned by $p_{i}$ and $q_{i}$ for $i=1,2$. When $L_{1}$ and $L_{2}$ are ultra-parallel, let $L_{\perp}$ be the complex line orthogonal to them. Let $z_{i}$ denote the point of intersection of $L_{\perp}$ and $L_{i}$ for $i=1,2$ and let $2 d$ denote the distance between $z_{1}$ and $z_{2}$. When $L_{1}$ and $L_{2}$ intersect in a single point, let $z_{1}=z_{2}$ denote this point of intersection and let $\phi$ denote the angle between $L_{1}$ and $L_{2}$. If $\mathbf{n}_{i}$ is a polar vector to $L_{i}$ normalised so that $\left\langle\mathbf{n}_{i}, \mathbf{n}_{i}\right\rangle=1$, then $\left|\left\langle\mathbf{n}_{2}, \mathbf{n}_{1}\right\rangle\right|$ equals $\cosh d$ or $\cos \phi$ in our two cases respectively; see page 100 of [5]. In order to not have to write out formulae in the separate cases we use the quantity $\left|\left\langle\mathbf{n}_{2}, \mathbf{n}_{1}\right\rangle\right|$, which we denote by $r$, as one of our coordinates. While doing so, we keep the geometrical significance of $r$ in mind.

The points $p_{i}, q_{i}$ and $z_{i}$ form a hyperbolic triangle in the natural Poincaré metric on $L_{i}$. Let $2 \theta_{i}$ be the angle at $z_{i}$ measured from the side joining $z_{i}, q_{i}$ to the side joining $z_{i}, p_{i}$; see Figure 2.1. This angle lies in the interval $(0, \pi)$. It is the internal angle of the triangle at $z_{i}$ if the triple $\left(z_{i}, q_{i}, p_{i}\right)$ is positively oriented with respect to the natural complex structure on $L_{i}$. Otherwise, it is the external angle. Drop a perpendicular from $z_{i}$ to the geodesic joining $p_{i}$ and $q_{i}$ and call it $\gamma_{i}$. Orient the geodesic containing $\gamma_{i}$ so that $\theta_{i}$ is the angle between its positive direction and the sides of the triangle with endpoint $z_{i}$. The length of $\gamma_{i}$ is related to $\theta_{i}$ by the angle of parallelism formulae; Theorem 7.9 of [1].

We now define one more parameter, an angle $\psi$ varying in $[0,2 \pi)$. In the ultra-parallel case, using parallel transport, move $\gamma_{2}$ along $L_{\perp}$ to $L_{1}$. Then $\psi$ is the angle in $L_{1}$ from the parallel transport of the positive direction of $\gamma_{2}$ to the positive direction of $\gamma_{1}$. In the spirit of the classical complex distance [8], [10], we use the following convention for the sign of $\psi$. When looking along the geodesic perpendicular to $L_{1}$ and $L_{2}$ the sign of $\psi$ should be positive; see Figure 2.1 and compare it with Figure 1 of [10]. In the case where $L_{1}$ and $L_{2}$ intersect in a point, we let $\Pi_{1}$ be orthogonal projection onto $L_{1}$. Then $\psi$ is defined to be the angle in $L_{1}$ measured from $\gamma_{1}$ to $\Pi_{1}\left(\gamma_{2}\right)$. Goldman 
calls this angle the phase; see Section 2.2.2 of [5]. Note that when $L_{1}$ and $L_{2}$ are orthogonal, that is $r=\left|\left\langle\mathbf{n}_{2}, \mathbf{n}_{1}\right\rangle\right|=0$, we have $\Pi_{1}\left(\gamma_{2}\right)=z_{1}$ and so $\psi$ is not defined. In fact, $r$ and $\psi$ form polar coordinates on $\mathbb{C}$.

In the degenerate case where $L_{1}=L_{2}$ we have $r=\left|\left\langle\mathbf{n}_{2}, \mathbf{n}_{1}\right\rangle\right|=1$. In this case we take $z_{1}=z_{2}$ to be any point of $L_{1}=L_{2}$ and we use it to define the other parameters $\theta_{1}, \theta_{2}$ and $\psi$ as above $(\psi$ is simply the angle between $\gamma_{1}$ and $\gamma_{2}$ ). By making a different choice of point $\tilde{z}_{1}=\tilde{z}_{2}$ we obtain different parameters $\tilde{\theta}_{1}, \tilde{\theta}_{2}$ and $\tilde{\psi}$. There is an equivalence relation relating two sets of parameters which correspond to the same four points $p_{i}$ and $q_{i}$ but to different choices of $z_{i}$ and $\tilde{z}_{i}$. We shall show that this leads to a collapse of the parameter space where $r=1$ to a 1-dimensional set. We give a geometrical interpretation of this collapse in the next section.

Writing $\mathbf{p}_{i}$ and $\mathbf{q}_{i}$ for lifts of $p_{i}$ and $q_{i}$ to $\mathbb{C}^{3}-\{0\}$, we define the three cross-ratios of our points $p_{i}$ and $q_{i}$ as follows:

$$
\begin{aligned}
& \mathbb{X}_{1}=\left[p_{2}, p_{1}, q_{1}, q_{2}\right]=\frac{\left\langle\mathbf{q}_{1}, \mathbf{p}_{2}\right\rangle\left\langle\mathbf{q}_{2}, \mathbf{p}_{1}\right\rangle}{\left\langle\mathbf{q}_{2}, \mathbf{p}_{2}\right\rangle\left\langle\mathbf{q}_{1}, \mathbf{p}_{1}\right\rangle}, \\
& \mathbb{X}_{2}=\left[p_{2}, q_{1}, p_{1}, q_{2}\right]=\frac{\left\langle\mathbf{p}_{1}, \mathbf{p}_{2}\right\rangle\left\langle\mathbf{q}_{2}, \mathbf{q}_{1}\right\rangle}{\left\langle\mathbf{q}_{2}, \mathbf{p}_{2}\right\rangle\left\langle\mathbf{p}_{1}, \mathbf{q}_{1}\right\rangle}, \\
& \mathbb{X}_{3}=\left[p_{1}, q_{1}, p_{2}, q_{2}\right]=\frac{\left\langle\mathbf{p}_{2}, \mathbf{p}_{1}\right\rangle\left\langle\mathbf{q}_{2}, \mathbf{q}_{1}\right\rangle}{\left\langle\mathbf{q}_{2}, \mathbf{p}_{1}\right\rangle\left\langle\mathbf{p}_{2}, \mathbf{q}_{1}\right\rangle},
\end{aligned}
$$

These cross-ratios are 0,1 or $\infty$ if and only if a pair of the points coincide. By hypothesis we exclude this possibility.

Our main theorem is that we can express these three cross-ratios in terms of our geometrical coordinates $r, \theta_{1}, \theta_{2}, \psi$.

Theorem 2.1. Let $p_{1}, q_{1}, p_{2}$ and $q_{2}$ be four pairwise distinct points of $\partial \mathbf{H}_{\mathbb{C}}^{2}$. Let $L_{i}$ be the complex line spanned by $p_{i}$ and $q_{i}$. Suppose that $L_{1}$ and $L_{2}$ are not asymptotic. Let $r, \theta_{1}, \theta_{2}$ and $\psi$ be the geometrical coordinates defined above. Then

$$
\begin{aligned}
& \mathbb{X}_{1}=\frac{r^{2} e^{i \theta_{1}+i \theta_{2}}-2 r \cos \psi+e^{-i \theta_{1}-i \theta_{2}}}{-4 \sin \theta_{1} \sin \theta_{2}}, \\
& \mathbb{X}_{2}=\frac{r^{2} e^{-i \theta_{1}+i \theta_{2}}-2 r \cos \psi+e^{i \theta_{1}-i \theta_{2}}}{4 \sin \theta_{1} \sin \theta_{2}}, \\
& \mathbb{X}_{3}=\frac{r^{2} e^{i \psi}-2 r \cos \left(\theta_{1}-\theta_{2}\right)+e^{-i \psi}}{r^{2} e^{i \psi}-2 r \cos \left(\theta_{1}+\theta_{2}\right)+e^{-i \psi}}
\end{aligned}
$$

Moreover, these expressions satisfy the cross-ratio identities (1.1), (1.2) and, when $r \neq 1$, the quantities $r e^{i \psi}, \theta_{1}$ and $\theta_{2}$ may be written uniquely in terms of $\mathbb{X}_{1}, \mathbb{X}_{2}$ and $\mathbb{X}_{3}$.

Finally, we consider the case where $L_{1}$ and $L_{2}$ are asymptotic. Again we have $r=\left|\left\langle\mathbf{n}_{2}, \mathbf{n}_{1}\right\rangle\right|=1$. Let $z_{1}=z_{2}$ be the intersection point of $\bar{L}_{1}$ and $\bar{L}_{2}$. The point $z_{i}$ now lies on $\partial L_{i}$ and so, provided it does not coincide with one our four original points, $z_{i}, p_{i}$ and $q_{i}$ are the vertices of an ideal triangle in $L_{i}$. This implies that $\theta_{1}$ and $\theta_{2}$ are each either 0 or $\pi$ and $\psi=\left|\theta_{1}-\theta_{2}\right|$. Hence both the numerator and the denominator of each of (2.1), (2.2) and (2.3) vanish. We use a blow-up at each of these points to evaluate $\mathbb{X}_{1}, \mathbb{X}_{2}$ and $\mathbb{X}_{3}$. In other words, we separate out the different tangent directions. Let $\left(r^{\prime}, \theta_{1}^{\prime}, \theta_{2}^{\prime}, \psi^{\prime}\right)$ be a tangent vector at one of the points where $r=1$, each $\theta_{i}$ is either 0 or $\pi$ and $\psi=\left|\theta_{1}-\theta_{2}\right|$. For each such tangent vector with $\theta_{i}^{\prime} \neq 0$ we can express $\mathbb{X}_{i}$ in terms of $r^{\prime}, \theta_{i}^{\prime}$ and $\psi^{\prime}$. 
Theorem 2.2. Let $p_{1}, q_{1}, p_{2}$ and $q_{2}$ be four pairwise distinct points of $\partial \mathbf{H}_{\mathbb{C}}^{2}$. Let $L_{i}$ be the complex line spanned by $p_{i}$ and $q_{i}$. Suppose that $L_{1}$ and $L_{2}$ are asymptotic at a point that is distinct from $p_{i}$ and $q_{i}$. Let $\left(r^{\prime}, \theta_{1}^{\prime}, \theta_{2}^{\prime}, \psi^{\prime}\right)$ be a tangent vector to the corresponding point of the space of coordinates defined above with $\theta_{i}^{\prime} \neq 0$. Then $r^{\prime} \neq 0$ and

$$
\begin{aligned}
& \mathbb{X}_{1}=\frac{r^{\prime 2}+2 i r^{\prime}\left(\theta_{1}^{\prime}+\theta_{2}^{\prime}\right)-\left(\theta_{1}^{\prime}+\theta_{2}^{\prime}\right)^{2}+\psi^{\prime 2}}{-4 \theta_{1}^{\prime} \theta_{2}^{\prime}}, \\
& \mathbb{X}_{2}=\frac{{r^{\prime}}^{2}-2 i r^{\prime}\left(\theta_{1}^{\prime}-\theta_{2}^{\prime}\right)-\left(\theta_{1}^{\prime}-\theta_{2}^{\prime}\right)^{2}+\psi^{\prime 2}}{4 \theta_{1}^{\prime} \theta_{2}^{\prime}}, \\
& \mathbb{X}_{3}=\frac{r^{\prime 2}-2 i r^{\prime} \psi^{\prime}-\psi^{\prime 2}+\left(\theta_{1}^{\prime}-\theta_{2}^{\prime}\right)^{2}}{r^{\prime 2}-2 i r^{\prime} \psi^{\prime}-\psi^{\prime 2}+\left(\theta_{1}^{\prime}+\theta_{2}^{\prime}\right)^{2}} .
\end{aligned}
$$

Moreover, these expressions satisfy the cross-ratio identities (1.1), (1.2) and $\theta_{1}^{\prime} / r^{\prime}, \theta_{2}^{\prime} / r^{\prime}$ and $\psi^{\prime} / r^{\prime}$ may be expressed uniquely in terms of $\mathbb{X}_{1}, \mathbb{X}_{2}$ and $\mathbb{X}_{3}$.

The last case to consider is when $L_{1}$ and $L_{2}$ are asymptotic at one of our four points. In what follows, we suppose that this point is $p_{2}$. The other cases are similar and we discuss them briefly in the final section. The points $p_{1}, q_{1}$ and $z_{1}=p_{2}$ again form an ideal triangle and so $\theta_{1}=0$ or $\pi$. Once again the denominator of (2.1) and (2.2) vanishes so we need to use a blow up. The difference is that this time we have $\theta_{1}^{\prime}=0$ as well. In order for $\mathbb{X}_{1}$ and $\mathbb{X}_{2}$ to be finite, their numerator must vanish as well. This means that we must use second derivatives of $r(t), \theta_{i}(t)$ and $\psi(t)$.

Theorem 2.3. Let $p_{1}, q_{1}, p_{2}$ and $q_{2}$ be four pairwise distinct points of $\partial \mathbf{H}_{\mathbb{C}}^{2}$. Let $L_{i}$ be the complex line spanned by $p_{i}$ and $q_{i}$. Suppose that $L_{1}$ and $L_{2}$ are asymptotic at $p_{2}$. Let $\left(r^{\prime}, \theta_{1}^{\prime}, \theta_{2}^{\prime}, \psi^{\prime}\right)$ be a tangent vector to the corresponding point of the space of coordinates. Then $\theta_{1}^{\prime}=0, r^{\prime}=0$ and $\psi^{\prime}=\theta_{2}^{\prime} \neq 0$. Let $\left(r^{\prime \prime}, \theta_{1}^{\prime \prime}, \theta_{2}^{\prime \prime}, \psi^{\prime \prime}\right)$ be the corresponding vector of second derivatives. Then

$$
\mathbb{X}_{1}=\frac{i r^{\prime \prime}-\theta_{1}^{\prime \prime}-\theta_{2}^{\prime \prime}+\psi^{\prime \prime}}{-2 \theta_{1}^{\prime \prime}}, \quad \mathbb{X}_{2}=\frac{i r^{\prime \prime}+\theta_{1}^{\prime \prime}-\theta_{2}^{\prime \prime}+\psi^{\prime \prime}}{2 \theta_{1}^{\prime \prime}}, \quad \mathbb{X}_{3}=\frac{i r^{\prime \prime}+\psi^{\prime \prime}+\theta_{1}^{\prime \prime}-\theta_{2}^{\prime \prime}}{i r^{\prime \prime}+\psi^{\prime \prime}-\theta_{1}^{\prime \prime}-\theta_{2}^{\prime \prime}} .
$$

In particular, $\mathbb{X}_{2}=1-\mathbb{X}_{1}$ and $\mathbb{X}_{3}=-\mathbb{X}_{2} / \mathbb{X}_{1}=1-1 / \mathbb{X}_{1}$.

\section{THE GENERIC CASES}

In this section we prove Theorem 2.1. We first treat the case where $L_{1}$ and $L_{2}$ are ultra-parallel and then the case where they intersect.

In the ultra-parallel case we normalise the four points $p_{1}, q_{1}, p_{2}$ and $q_{2}$ in terms of the parameters $d, \theta_{1}, \theta_{2}$ and $\psi$ as follows. We do this both in terms of Heisenberg coordinates on $\partial \mathbf{H}_{\mathbb{C}}^{2}$ and as lifts of these points to $\mathbb{C}^{3}$. We begin by supposing that $\partial L_{\perp}$ is the vertical axis in the Heisenberg group. A complex line $L$ is orthogonal to $L_{\perp}$ if and only if $\partial L$ is a Euclidean circle centred on the vertical axis. We choose $\partial L_{1}$ to be the circle of radius 1 and height 0 . Then the points $p_{1}$ and $q_{1}$ may be taken to be any points on this circle subtending an angle $2 \theta_{1}$ at the centre. We take them to be

$$
p_{1}=\left(e^{i \theta_{1}}, 0\right), \quad q_{2}=\left(e^{-i \theta_{1}}, 0\right) .
$$

We choose $\partial L_{2}$ to be another circle of height 0 . The fact that the distance between $L_{1}$ and $L_{2}$ is $2 d$ implies that the radius of $\partial L_{2}$ is $e^{d}$. Again, $p_{2}$ and $q_{2}$ are points on this circle subtending an angle of $2 \theta_{2}$. The angle $\psi$ is the angle between the centre of this interval and that of the interval between $p_{1}$ and $q_{1}$. We must be careful about the direction of $\psi$; see Figure 2.1. Since $e^{d}>1$, when looking along the geodesic from $z_{1}$ to $z_{2}$ a positive angle $\psi$ is negative with respect to the natural orientation on $L_{2}$. Thus we have

$$
p_{2}=\left(e^{d+i \theta_{2}-i \psi}, 0\right) \quad q_{2}=\left(e^{d-i \theta_{2}-i \psi}, 0\right) .
$$


To calculate cross-ratios it is convenient to take the standard lifts of these four points. They are

$$
\mathbf{p}_{1}=\left[\begin{array}{c}
-1 \\
\sqrt{2} e^{i \theta_{1}} \\
1
\end{array}\right], \quad \mathbf{q}_{1}=\left[\begin{array}{c}
-1 \\
\sqrt{2} e^{-i \theta_{1}} \\
1
\end{array}\right], \quad \mathbf{p}_{2}=\left[\begin{array}{c}
-e^{2 d} \\
\sqrt{2} e^{d+i \theta_{2}-i \psi} \\
1
\end{array}\right], \quad \mathbf{q}_{2}=\left[\begin{array}{c}
-e^{2 d} \\
\sqrt{2} e^{d-i \theta_{2}-i \psi} \\
1
\end{array}\right] .
$$

Substituting the vectors (3.3) into (2.1) gives:

$$
\begin{aligned}
\mathbb{X}_{1}= & \frac{\left\langle\mathbf{q}_{1}, \mathbf{p}_{2}\right\rangle\left\langle\mathbf{q}_{2}, \mathbf{p}_{1}\right\rangle}{\left\langle\mathbf{q}_{2}, \mathbf{p}_{2}\right\rangle\left\langle\mathbf{q}_{1}, \mathbf{p}_{1}\right\rangle} \\
& \left\langle\left[\begin{array}{c}
-1 \\
\sqrt{2} e^{-i \theta_{1}} \\
1
\end{array}\right],\left[\begin{array}{c}
-e^{2 d} \\
\sqrt{2} e^{d+i \theta_{2}-i \psi} \\
1
\end{array}\right]\right\rangle\left\langle\left[\begin{array}{c}
-e^{2 d} \\
\sqrt{2} e^{d-i \theta_{2}-i \psi} \\
1
\end{array}\right],\left[\begin{array}{c}
-1 \\
\sqrt{2} e^{i \theta_{1}} \\
1
\end{array}\right]\right\rangle \\
& \left\langle\left[\begin{array}{c}
-e^{2 d} \\
\sqrt{2} e^{d-i \theta_{2}-i \psi} \\
1
\end{array}\right],\left[\begin{array}{c}
-e^{2 d} \\
\sqrt{2} e^{d+i \theta_{2}-i \psi} \\
1
\end{array}\right]\right\rangle\left\langle\left[\begin{array}{c}
-1 \\
\sqrt{2} e^{-i \theta_{1}} \\
1
\end{array}\right],\left[\begin{array}{c}
-1 \\
\sqrt{2} e^{i \theta_{1}} \\
1
\end{array}\right]\right\rangle \\
= & \frac{\cosh ^{2} d e^{i \theta_{1}+i \theta_{2}}-2 \cosh d \cos \psi+e^{-i \theta_{1}-i \theta_{2}}}{-4 \sin \theta_{1} \sin \theta_{2}} .
\end{aligned}
$$

This has proved (2.4). Substituting (3.3) into (2.2) and (2.3) enables us to prove (2.5) and (2.6) similarly:

$$
\begin{aligned}
& \mathbb{X}_{2}=\frac{\left\langle\mathbf{p}_{1}, \mathbf{p}_{2}\right\rangle\left\langle\mathbf{q}_{2}, \mathbf{q}_{1}\right\rangle}{\left\langle\mathbf{q}_{2}, \mathbf{p}_{2}\right\rangle\left\langle\mathbf{p}_{1}, \mathbf{q}_{1}\right\rangle}=\frac{\cosh ^{2} d e^{-i \theta_{1}+i \theta_{2}}-2 \cosh d \cos \psi+e^{i \theta_{1}-i \theta_{2}}}{4 \sin \theta_{1} \sin \theta_{2}}, \\
& \mathbb{X}_{3}=\frac{\left\langle\mathbf{p}_{2}, \mathbf{p}_{1}\right\rangle\left\langle\mathbf{q}_{2}, \mathbf{q}_{1}\right\rangle}{\left\langle\mathbf{q}_{2}, \mathbf{p}_{1}\right\rangle\left\langle\mathbf{p}_{2}, \mathbf{q}_{1}\right\rangle}=\frac{\cosh ^{2} d e^{i \psi}-2 \cosh d \cos \left(\theta_{1}-\theta_{2}\right)+e^{-i \psi}}{\cosh ^{2} d e^{i \psi}-2 \cosh d \cos \left(\theta_{1}+\theta_{2}\right)+e^{-i \psi}}
\end{aligned}
$$

The details of the case where $L_{1}$ and $L_{2}$ intersect are very similar and we leave them to the reader. In this case we have $r=\cos \phi<1$ and, using the ball model, we normalise so that

$$
\begin{array}{ll}
p_{1}=\left(e^{i \theta_{1}}, 0\right), & q_{1}=\left(e^{-i \theta_{1}}, 0\right), \\
p_{2}=\left(r e^{i \psi+i \theta_{2}}, \sqrt{1-r^{2}} e^{i \theta_{2}}\right), & q_{2}=\left(r e^{i \psi-i \theta_{2}}, \sqrt{1-r^{2}} e^{-i \theta_{2}}\right) .
\end{array}
$$

Lifting these points to $\mathbb{C}^{3}$ and substituting them into (2.1), (2.2) and (2.3) gives the expressions (2.4), (2.5) and (2.6).

When $L_{1}=L_{2}$, that is $r=1$, we choose $z_{1}=z_{2}$ to be any point of $L_{1}=L_{2}$. Then we may use the same formulae as in intersecting case to find expressions for $\mathbb{X}_{1}, \mathbb{X}_{2}$ and $\mathbb{X}_{3}$. Namely:

$$
\mathbb{X}_{1}=\frac{\cos \left(\theta_{1}+\theta_{2}\right)-\cos \psi}{-2 \sin \theta_{1} \sin \theta_{2}}, \quad \mathbb{X}_{2}=\frac{\cos \left(\theta_{1}-\theta_{2}\right)-\cos \psi}{2 \sin \theta_{1} \sin \theta_{2}}, \quad \mathbb{X}_{3}=\frac{\cos \psi-\cos \left(\theta_{1}-\theta_{2}\right)}{\cos \psi-\cos \left(\theta_{1}+\theta_{2}\right)} .
$$

Hence, it is clear that the $\mathbb{X}_{i}$ are real and satisfy $\mathbb{X}_{2}=1-\mathbb{X}_{1}$ and $\mathbb{X}_{3}=-\mathbb{X}_{2} / \mathbb{X}_{1}=1-1 / \mathbb{X}_{1}$ and the cross-ratio variety has collapsed to a 1-dimensional set. These are the identities satisfied by the classical cross-ratio; see page 76 of [1]. As we discussed above, making a different choice of $z_{1}=z_{2}$ yields different parameters, also satisfying the above identities. We now interpret this geometrically. Let $\alpha_{i}$ be the geodesic joining $p_{i}$ and $q_{i}$. If $\alpha_{1}$ and $\alpha_{2}$ intersect let $\beta$ be the angle between them. If $\alpha_{1}$ and $\alpha_{2}$ are ultra-parallel let $b$ be the distance between them. The parameters $\beta$ and $b$ are independent of our choice of $z_{1}=z_{2}$. The geodesics $\gamma_{i}$ and $\alpha_{i}$ (together with their common perpendicular if appropriate) form a hyperbolic quadrilateral or pentagon. Using hyperbolic trigonometry we can show that our coordinates give us $\beta$ or $b$ respectively in terms of $\mathbb{X}_{1}$. Namely, if $c_{i}$ is the length of $\gamma_{i}$, then the angle of parallelism formulae imply:

$$
1-2 \mathbb{X}_{1}=\frac{\cos \theta_{1} \cos \theta_{2}-\cos \psi}{\sin \theta_{1} \sin \theta_{2}}=\sinh c_{1} \sinh c_{2}-\cos \psi \cosh c_{1} \cosh c_{2}
$$


Thus, using the identities in sections VI.3.4 and VI.3.2 of [4], we see that $1-2 \mathbb{X}_{1}=\cos \beta$ or $1-2 \mathbb{X}_{1}=\cosh b$ respectively. These identities are satisfied by the classical cross-ratio (see sections 7.24 and 7.23 of [1]) and this shows that, in this case, the Korányi-Reimann cross-ratio and the classical one coincide. The parameters $\theta_{1}, \theta_{2}, \psi$ and $\tilde{\theta}_{1}, \tilde{\theta}_{2}, \tilde{\psi}$ are equivalent if and only if they determine quadrilaterals or pentagons with the same angle $\beta$ or side length $b$.

We now show that, in the case where $r \neq 1$, that we may solve for $r e^{i \psi}, \theta_{1}$ and $\theta_{2}$ in terms of $\mathbb{X}_{1}, \mathbb{X}_{2}$ and $\mathbb{X}_{3}$. From (2.4), (2.5) and (2.6) we obtain

$$
\begin{aligned}
& 2 \Re\left(\mathbb{X}_{1}+\mathbb{X}_{2}\right)=r^{2}+1, \\
& 2 \Im\left(\mathbb{X}_{1}+\mathbb{X}_{2}\right)=-\left(r^{2}-1\right) \cot \theta_{2}=-\left(2 \Re\left(\mathbb{X}_{1}+\mathbb{X}_{2}\right)-2\right) \cot \theta_{2}, \\
& 2 \Im\left(\mathbb{X}_{1}-\mathbb{X}_{2}\right)=-\left(r^{2}-1\right) \cot \theta_{1}=-\left(2 \Re\left(\mathbb{X}_{1}+\mathbb{X}_{2}\right)-2\right) \cot \theta_{1}, \\
& 2 \Re\left(\mathbb{X}_{1}-\mathbb{X}_{2}\right)=-\left(r^{2}+1\right) \cot \theta_{1} \cot \theta_{2}+2 r \csc \theta_{1} \csc \theta_{2} \cos \psi .
\end{aligned}
$$

The first three of these expressions enable us to express $r^{2}$, $\cot \theta_{1}$ and $\cot \theta_{2}$ in terms of $\mathbb{X}_{1}$ and $\mathbb{X}_{2}$ (for the last two we use $r^{2} \neq 1$ ). Since $r \geq 0$ and $\theta_{i} \in(0, \pi)$ this determines $r, \theta_{1}$ and $\theta_{2}$. When $r=0$ we have already explained why $\psi$ is undefined. When $r \neq 0$ the last equation enables us to express $\cos \psi$ in terms of the other variables. Since $\psi$ varies in $[0,2 \pi)$ this does not determine $\psi$ uniquely. In order to do so, we must use $\mathbb{X}_{3}$ to find $\sin \psi$ : When $r \neq 0$, we have

$$
\Im\left(\frac{1}{1-\mathbb{X}_{3}}\right)=\Im\left(\frac{r^{2} e^{i \psi}-2 r \cos \left(\theta_{1}+\theta_{2}\right)+e^{-i \psi}}{4 r \sin \theta_{1} \sin \theta_{2}}\right)=\frac{\left(r^{2}-1\right) \sin \psi}{4 r \sin \theta_{1} \sin \theta_{2}} .
$$

Thus we can write $r e^{i \psi}$ as a function of $\mathbb{X}_{1}, \mathbb{X}_{2}$ and $\mathbb{X}_{3}$.

To complete the proof of Theorem 2.1, we now verify that our expressions for $\mathbb{X}_{1}, \mathbb{X}_{2}$ and $\mathbb{X}_{3}$ satisfy (1.1) and (1.2). From (2.4) we have:

$$
\begin{aligned}
\left|\mathbb{X}_{1}\right|^{2} & =\frac{\left(r^{2}-1\right)^{2}-4 r\left(r^{2}+1\right) \cos \left(\theta_{1}+\theta_{2}\right) \cos \psi+4 r^{2}\left(\cos ^{2}\left(\theta_{1}+\theta_{2}\right)+\cos ^{2} \psi\right)}{16 \sin ^{2} \theta_{1} \sin ^{2} \theta_{2}} \\
\left|\mathbb{X}_{2}\right|^{2} & =\frac{\left(r^{2}-1\right)^{2}-4 r\left(r^{2}+1\right) \cos \left(\theta_{1}-\theta_{2}\right) \cos \psi+4 r^{2}\left(\cos ^{2}\left(\theta_{1}-\theta_{2}\right)+\cos ^{2} \psi\right)}{16 \sin ^{2} \theta_{1} \sin ^{2} \theta_{2}} \\
\left|\mathbb{X}_{3}\right|^{2} & =\frac{\left(r^{2}-1\right)^{2}-4 r\left(r^{2}+1\right) \cos \left(\theta_{1}-\theta_{2}\right) \cos \psi+4 r^{2}\left(\cos ^{2}\left(\theta_{1}-\theta_{2}\right)+\cos ^{2} \psi\right)}{\left(r^{2}-1\right)^{2}-4 r\left(r^{2}+1\right) \cos \left(\theta_{1}+\theta_{2}\right) \cos \psi+4 r^{2}\left(\cos ^{2}\left(\theta_{1}+\theta_{2}\right)+\cos ^{2} \psi\right)}
\end{aligned}
$$

This immediately yields the identity $\left|\mathbb{X}_{3}\right|=\left|\mathbb{X}_{2}\right| /\left|\mathbb{X}_{1}\right|$, which is (1.1). Using the expressions derived above and simplifying, we have:

$$
\begin{aligned}
2\left|\mathbb{X}_{1}\right|^{2} \Re\left(\mathbb{X}_{3}\right) \\
=\frac{2 \Re\left(\left(r^{2} e^{i \psi}-2 r \cos \left(\theta_{1}-\theta_{2}\right)+e^{-i \psi}\right)\left(r^{2} e^{-i \psi}-2 r \cos \left(\theta_{1}+\theta_{2}\right)+e^{i \psi}\right)\right)}{16 \sin ^{2} \theta_{1} \sin ^{2} \theta_{2}} \\
=\frac{\left(r^{2}-1\right)^{2}-4 r\left(r^{2}+1\right) \cos \left(\theta_{1}+\theta_{2}\right) \cos \psi+4 r^{2} \cos ^{2} \psi+4 r^{2} \cos \left(\theta_{1}+\theta_{2}\right) \cos \left(\theta_{1}-\theta_{2}\right)}{16 \sin ^{2} \theta_{1} \sin ^{2} \theta_{2}} \\
\quad+\frac{\left(r^{2}-1\right)^{2}-4 r\left(r^{2}+1\right) \cos \left(\theta_{1}-\theta_{2}\right) \cos \psi+4 r^{2} \cos ^{2} \psi+4 r^{2} \cos \left(\theta_{1}+\theta_{2}\right) \cos \left(\theta_{1}-\theta_{2}\right)}{16 \sin ^{2} \theta_{1} \sin ^{2} \theta_{2}} \\
=\left|\mathbb{X}_{1}\right|^{2}+\left|\mathbb{X}_{2}\right|^{2}-2 \Re\left(\mathbb{X}_{1}+\mathbb{X}_{2}\right)+1 .
\end{aligned}
$$

This verifies (1.2) as required. 


\section{The ASYMPtotic CASE}

In this section we consider the case where $L_{1}$ and $L_{2}$ are asymptotic and our goal is to prove Theorems 2.2 and 2.3 .

Since $L_{1}$ and $L_{2}$ are asymptotic, we have $r=1$. Assume first that the point $z_{1}=z_{2}$ which is $\partial L_{1} \cap \partial L_{2}$ is distinct from $p_{i}$ and $q_{i}$. In this case $z_{i}, p_{i}$ and $q_{i}$ form an ideal triangle. The internal angle of this triangle at $z_{i}$ is 0 . Thus $\theta_{i}=0$ or $\theta_{i}=\pi$ depending on the orientation of $\gamma_{i}$. The tangent vectors to $\gamma_{i}$ at $z_{i}$ are either equal or opposite and so $\psi=0$ or $\pi$ respectively. In other words, $\psi=\left|\theta_{1}-\theta_{2}\right|$. Hence the numerator and denominator in each of (2.1), (2.2) and (2.3) vanish.

Consider a path parametrised by $t$ that is given by twice differentiable real valued functions $r=r(t), \theta_{i}=\theta_{i}(t)$ and $\psi=\psi(t)$. Suppose that $\theta_{i}(t) \in(0, \pi)$ for $t \neq 0$ and also $r(0)=1$, each $\theta_{i}(0)$ is either 0 or $\pi$ and $\psi(0)=\left|\theta_{1}(0)-\theta_{2}(0)\right|$. We evaluate the $\mathbb{X}_{i}$ by substituting these values into our expressions from Theorem 2.1 and letting $t$ tend to zero. Geometrically this is the same as a blow-up. We write $r^{\prime}(t), \theta_{i}^{\prime}(t)$ and $\psi^{\prime}(t)$ for the derivatives. For ease of notation, we write $r^{\prime}$, $\theta_{i}^{\prime}$ and $\psi^{\prime}$ for $r^{\prime}(0), \theta_{i}^{\prime}(0)$ and $\psi^{\prime}(0)$ respectively. By reparametrising if necessary, we may suppose that $\left(r^{\prime}, \theta_{1}^{\prime}, \theta_{2}^{\prime}, \psi^{\prime}\right)$ is non-trivial and so is the tangent vector to our path at $t=0$. Using l'Hôpital's rule twice on each of (2.1), (2.2) and (2.3) yields (2.7), (2.8) and (2.9). If $r^{\prime}=0$ then $r=1$ for all points of our path. In other words, since $\theta_{i}(t) \in(0, \pi)$ for $t \neq 0$, we have $L_{1}=L_{2}$ when $t \neq 0$ and hence, by continuity, $L_{1}=L_{2}$ when $t=0$ as well. This can be seen directly by observing that the $\mathbb{X}_{i}$ are all real and satisfy $\mathbb{X}_{2}=1-\mathbb{X}_{1}$ and $\mathbb{X}_{3}=-\mathbb{X}_{2} / \mathbb{X}_{1}=1-1 / \mathbb{X}_{1}$. Use of Proposition 4.14 of [9] implies that $L_{1}=L_{2}$.

The effect of changing $\theta_{1}$ from 0 to $\pi$ or vice versa while fixing $\theta_{2}$ (and so changing $\psi=\left|\theta_{1}-\theta_{2}\right|$ as appropriate) is to multiply the numerator and denominator of each of the $\mathbb{X}_{i}$ by -1 . Hence, the formulae (2.7), (2.8) and (2.9) are the same for each choice of $\theta_{i} \in\{0, \pi\}$ with $\psi=\left|\theta_{1}-\theta_{2}\right|$. Suppose that we are given $\theta_{1}^{\prime}>0$. Since $\theta_{1}(t) \in(0, \pi)$ for $t \neq 0$ this is is the limit as $t$ tends to zero from above of points where $\theta_{1}(t)$ tends to 0 but is the limit as $t$ tends to 0 from below of points where $\theta_{2}(t)$ tends to $\pi$; that is, points approximated by $\theta_{1}(t)=t \theta_{1}^{\prime}$ for $t>0$ and $\theta_{1}^{\prime}(t)=\pi+t \theta_{1}^{\prime}$ for $t<0$. Similar arguments apply to the case of $\theta_{1}^{\prime}<0, \theta_{2}^{\prime}>0$ and $\theta_{2}^{\prime}<0$. This leads to an identification of each tangent direction to the points $\left(r, \theta_{1}, \theta_{2}, \psi\right)=(1,0,0,0)$ and $(1, \pi, \pi, 0)$ and the points $(1, \pi, 0, \pi)$ and $(1,0, \pi, \pi)$.

We can verify (2.7), (2.8) and (2.9) directly as follows. Normalise so that $L_{1}$ and $L_{2}$ are asymptotic at $z_{1}=z_{2}=\infty$ on the boundary of the Siegel domain and then take $p_{i}$ and $q_{i}$ to be points in the Heisenberg group with the following coordinates

$$
p_{1}=\left(0, \theta_{1}^{\prime}\right), \quad q_{1}=\left(0,-\theta_{1}^{\prime}\right), \quad p_{2}=\left(\sqrt{r^{\prime}}, \theta_{2}^{\prime}+\psi^{\prime}\right), \quad q_{2}=\left(\sqrt{r^{\prime}},-\theta_{2}^{\prime}+\psi^{\prime}\right) .
$$

Lifting these points to vectors in $\mathbb{C}^{3}$ and evaluating directly gives (2.7), (2.8) and (2.9).

As in the generic case, by writing:

$$
\Re\left(\mathbb{X}_{1}+\mathbb{X}_{2}\right)=1, \quad \Im\left(\mathbb{X}_{1}+\mathbb{X}_{2}\right)=\frac{r^{\prime}}{-\theta_{2}^{\prime}}, \quad \Im\left(\mathbb{X}_{1}-\mathbb{X}_{2}\right)=\frac{r^{\prime}}{-\theta_{1}^{\prime}}, \quad \Im\left(\frac{1}{1-\mathbb{X}_{3}}\right)=\frac{-r^{\prime} \psi^{\prime}}{2 \theta_{1}^{\prime} \theta_{2}^{\prime}},
$$

it is obvious that we can completely determine $\theta_{1}^{\prime} / r^{\prime}, \theta_{2}^{\prime} / r^{\prime}$ and $\psi^{\prime} / r^{\prime}$ from $\mathbb{X}_{1}, \mathbb{X}_{2}$ and $\mathbb{X}_{3}$. Furthermore, it is straightforward to verify that (2.7), (2.8) and (2.9) satisfy (1.1) and (1.2). This completes the proof of Theorem 2.2.

We now suppose that one of the $\theta_{i}^{\prime}$ is zero. We will show that, in this case, the point $z_{1}=z_{2}$ at which $L_{1}$ and $L_{2}$ are asymptotic must be one of the $p_{i}$ or the $q_{i}$. Suppose that $\theta_{1}^{\prime}=0$ but yet $\mathbb{X}_{i}$ are each finite. Since the denominators of $\mathbb{X}_{1}$ and $\mathbb{X}_{2}$ vanish, so must the numerators. Hence, we must have $r^{\prime 2}+2 i r^{\prime} \theta_{2}^{\prime}-\theta_{2}^{\prime 2}+\psi^{\prime 2}=0$. If $\theta_{2}^{\prime}=0$ then $r^{\prime}=0$ and $\psi^{\prime}=0$ which we have supposed to not be the case. So we assume that $\theta_{2}^{\prime} \neq 0$, and so $r^{\prime}=0$ and $\psi^{\prime}= \pm \theta_{2}^{\prime}$. We take the plus sign. 
Then the expressions (2.10) follow by applying l'Hôpitals's rule to (2.7), (2.8) and (2.9). From these expressions it is easy to see that $\mathbb{X}_{2}=1-\mathbb{X}_{1}$ and $\mathbb{X}_{3}=-\mathbb{X}_{2} / \mathbb{X}_{1}=1-1 / \mathbb{X}_{1}$. Using Proposition 4.7 of [9] we see that the angular invariant of $p_{1}, q_{1}$ and $p_{2}$ is $\pm \pi / 2$ and so these three points all lie on the boundary of the same complex geodesic, namely $L_{1}$. In other words, $L_{1}$ and $L_{2}$ are asymptotic at $p_{2}$.

Conversely, for any real numbers $r^{\prime \prime} / \theta_{1}^{\prime \prime}$ and $\left(\psi^{\prime \prime}-\theta_{2}^{\prime \prime}\right) / \theta_{1}^{\prime \prime}$ the expressions $(2.10)$ are realised by taking

$$
p_{1}=\left(0, \theta_{1}^{\prime \prime}\right), \quad q_{1}=\left(0,-\theta_{1}^{\prime \prime}\right), \quad p_{2}=\infty, \quad q_{2}=\left(\sqrt{r^{\prime \prime}},-\theta_{2}^{\prime \prime}+\psi^{\prime \prime}\right) .
$$

When $r^{\prime \prime} \neq 0$ the point $q_{2}$ does not lie on $\partial L_{1}$ and so $L_{1}$ and $L_{2}$ are asymptotic at $p_{2}$.

If we had chosen $\theta_{1}^{\prime}=0$ and $\psi^{\prime}=-\theta_{2}^{\prime} \neq 0$ then we would have had $\mathbb{X}_{2}=1-\mathbb{X}_{1}$ and $\mathbb{X}_{3}=-\overline{\mathbb{X}}_{2} / \overline{\mathbb{X}}_{1}=1-1 / \overline{\mathbb{X}}_{1}$. This would have lead to the case where $L_{1}$ and $L_{2}$ were asymptotic at $q_{2}$. Likewise, the cases where $\theta_{2}^{\prime}=0$ and $\psi^{\prime}= \pm \theta_{1}^{\prime} \neq 0$ correspond to the cases where $L_{1}$ and $L_{2}$ are asymptotic at $p_{1}$ or $q_{1}$.

\section{REFERENCES}

[1] A.F. Beardon; The Geometry of Discrete Groups. Graduate Texts in Mathematics 91, Springer (1983).

[2] E. Falbel; Geometric structures associated to triangulations as fixed point sets of involutions. Preprint 2005.

[3] E. Falbel \& I.D. Platis; The cross-ratio variety. In preparation.

[4] W. Fenchel; Elementary Geometry in Hyperbolic Space. de Gruyter Studies in Mathematics 11 (1989).

[5] W.M. Goldman; Complex Hyperbolic Geometry. Oxford Mathematical Monographs. Oxford University Press (1999).

[6] Y. Jiang, S. Kamiya \& J.R. Parker; Jørgensen's inequality for complex hyperbolic space. Geometriae Dedicata 97 (2003) 55-80.

[7] A. Korányi \& H.M. Reimann; The complex cross-ratio on the Heisenberg group. L'Enseign. Math. 33 (1987) 291-300.

[8] C. Kourouniotis; Complex length coordinates for quasi-Fuchsian groups. Mathematika 41 (1994) $173-188$.

[9] J.R. Parker \& I.D. Platis; Complex hyperbolic Fenchel-Nielsen coordinates. Preprint 2005.

[10] J.R. Parker \& C. Series; Bending formulae for convex hull boundaries. J. d'Analyse Math. 67 (1995) 165-198.

[11] P. Will; Groupes libres, groupes triangulaires et tore épointé dans PU(2,1). Ph.D. thesis University of Paris VI (2006).

Department of Mathematical Sciences, University of Durham, Durham DH1 3LE, United Kingdom, Department of Mathematics, Aristotle University of Salonica, Salonica, Greece

E-mail address: j.r.parker@dur.ac.uk, johnny-platis@yahoo.com.au 